Rapid Reviews COVID-19

\title{
Review 2: "Evaluation of a Machine Learning Approach Utilizing Wearable Data for Prediction of SARS-CoV-2 Infection in Healthcare Workers"
}

\section{Toyya Pujol ${ }^{1}$}

${ }^{1}$ RAND Corporation

Published on: Jan 26, 2022

License: Creative Commons Attribution 4.0 International License (CC-BY 4.0). 


\section{RR:C19 Evidence Scale rating by reviewer:}

- Potentially informative. The main claims made are not strongly justified by the methods and data, but may yield some insight. The results and conclusions of the study may resemble those from the hypothetical ideal study, but there is substantial room for doubt. Decision-makers should consider this evidence only with a thorough understanding of its weaknesses, alongside other evidence and theory. Decisionmakers should not consider this actionable, unless the weaknesses are clearly understood and there is other theory and evidence to further support it.

$* * * * * * * * * * * * * * * * * * * * * * * * * * * * * * * * * * * * * * * *$

\section{Review:}

This preprint, by Hirten et al, presents a study in which heart rate variation (HRV) was used to predict COVID-19 status of healthcare workers. Previous work, mentioned in the article, demonstrate that data from wearables has been successful in predicting COVID-19 status, in some cases even before patients are symptomatic. The study fits well into existing work in the area by building off previous work of wearables and changing in heart rate (such as the daily amplitude) can help predict COVID status. However, these other studies included more information than just heart rate based variables and very basic demographics (age and gender). The authors state that the main contribution of this study is training the model and using testing data to determine accuracy.

The study included over 400 health care workers with each participant's heart rate being collected using apple watches for a median of 73 days. The authors address the low number of positive COVID cases (12\%) in their analysis but do not discuss anything regarding the study's enrollment bias or the disproportionate number of women in the study. A major weakness in the article is that the sampling method is not clear to the reader. The authors state: "Data was split into independent training and testing sets, ensuring that observations were taken on chronologically similar days (e.g., Day 6 and Day 7), for the same subject, were in the same set. A sampling procedure was employed that ensured that observations with proximity in time $( \pm 4$ days), for the same subject, did not appear in both training and testing sets." This can be interpreted as: multiple observations of the same subject can appear in the same set if the observations are chronically close and that the same subject may appear in both the training and testing sets if the observations are chronically far apart. Having 
multiple observations from the same subject creates problems because the observations would be correlated. There are methods that can be used to address correlation in tree-based methods [1] but they were not mentioned in the paper. If the same subject is in both the training and the testing set, then the two sets are not independent which can result in a falsely high prediction rate. Furthermore, the fact that COVID positive patients are less likely to contract COVID again for some period of time after recovery presents additional correlation issues in which the \pm 4 days window would not be sufficient to claim the sets are independent. If this is indeed the analysis performed by the authors, then the method used is inappropriate for the type of data used in the study. The assumptions for GBM were violated. However, if this was not the case, the language should be corrected and expanded to avoid confusion. In addition, the authors would need to address how the issue of correlated data was handled or why in this case it can be ignored.

Given the unaddressed limitations of the analysis, the results from the testing data is suggestive and do not support the claim that the HRV measured by a wearable device can reliably predict COVID infections. It is these reasons that the authors' claim of the article's contribution of using testing data is further weakened. This study has an interesting idea but some additional work is needed to better understand the predictive power of HRV on COVID-19 status.

1. Rabinowicz A, Rosset S. Trees-Based Models for Correlated Data. arXiv preprint arXiv:2102.08114. 2021 Feb 16. 